\title{
Fuzzy Rule Based Image Reconstruction for PET
}

\author{
Partha P. Mondal \\ Department of Physics \\ Indian Institute of Science \\ Bangalore, India \\ partha@physics.iisc.ernet.in
}

\author{
K. Rajan \\ Department of Physics \\ Indian Institute of Science \\ Bangalore, India \\ rajan@physics.iisc.ernet.in
}

\begin{abstract}
Emission tomography imaging modality has given a new dimension to the field of medicine and biology. The maximum a-posteriori (MAP) and maximum likelihood (MI) algorithms are the widely used reconstruction algorithms for emission tomography. However, the images reconstructed by $M A P$ and $M L$ methods still suffer from artifacts such as noise, over-smoothing and streaking artifacts. These algorithms often fail to recognize the density class in the reconstruction and hence result in overpenalization causing blurring effect. A good knowledge of prior distribution is a must for MAP-based method. Recently proposed median root prior (MRP) algorithm preserves the edges in the image, but the reconstructed image suffers from step like streaking artifact. In this work, a fuzzy logic based approach is proposed for the pixel-pixel nearest neighborhood interaction. The proposed algorithm consists of two elementary steps: (1) Edge detection - fuzzy rule based derivatives are used for the detection of edges in the nearest neighborhood window. (2) Fuzzy smoothing penalization is performed only for those pixels for which edges are missing in the neighborhood window. Analysis shows that the proposed fuzzy rule based reconstruction algorithm is capable of producing better estimates compared to the images reconstructed by MAP and MRP algorithms. The reconstructed images are sharper with small features being better resolved due to the nature of the fuzzy potential function.
\end{abstract}

Keywords: Fuzzy Potential, Image Reconstruction, Maximum A-Posteriori Estimation, Maximum Likelihood, Positron Emission Tomography.

\section{Introduction}

Penalized image reconstruction algorithms are central to image reconstruction in emission tomography (ET). A complete and descriptive understanding of iterative image reconstruction algorithms for emission tomography (ET) can be found in $[1,2]$. Good reconstruction demands large computational time and the knowledge of prior distribution. The iterative algorithms like, maximum likelihood (ML) $[1,2]$, maximum a-posteriori (MAP) $[3,4,5]$, and MRP [6,7] are capable of generating good quality images at the cost of artifacts like noise, over-smoothness and streaking effect [6]. MAP-estimation involves controlling the desired features of the solution (reconstructed image) via parameters of prior distribution. The prior knowledge (which is contained in the penalty term) helps in producing the desired effect in the reconstructed image. For example, smooth priors produce smooth reconstruction $[3,4,5]$ where as edge-preserving priors $[6,7]$ produce sharp reconstruction.

Fuzzy techniques have been successfully applied in image processing applications such as image restoration [9], and interpolation [10]. In the present work we have extended fuzzy concepts to image reconstruction for ET. The prior distribution is defined by Gibbs distribution and the potential (which defines the nature of nearest neighbor interactions) is modeled using fuzzy rules. Proposed fuzzy rule based potential consists of two major operations: fuzzy filtering followed by fuzzy smoothing. These operations are performed iteratively until the estimate converges and stabilizes.

\section{Reconstruction Algorithms for PET}

The measurements in PET, $y_{m}, m=1, \ldots, M$ are modeled as independent Poisson random variables with mean $\sum_{n=1}^{N} \lambda_{n} p_{n m}, m=1, \ldots, M$ where, $\lambda_{n}, n=1, \ldots, N$ are the mean parameters of the emission process and $p_{n m}$ is the probability that an annihilation in the $n^{\text {th }}$ pixel is detected in $m^{\text {th }}$ detector. The likelihood function i.e., the conditional probability for observing $\mathrm{Y}=\mathrm{y}$ given the emission parameter $\Lambda=\lambda$ is the joint probability of the individual Poisson process i.e,

$$
P(y / \lambda)=\prod_{m=1}^{M}\left[\frac{\exp \left(-\sum_{n=1}^{N} \lambda_{n} p_{n m}\right)\left(-\sum_{n=1}^{N} \lambda_{n} p_{n m}\right)^{y_{m}}}{y_{m} !}\right]
$$

Reconstruction algorithm proceeds by finding the estimate $\lambda$, which maximizes the objective function. In maximum a-posteriori (MAP) estimation, the objective function is taken as the posterior distribution function. The task is the determination of the estimate $\lambda$, which

\footnotetext{
${ }^{*}$ 0-7803-8566-7/04/\$20.00 @ 2004 IEEE.
} 
maximizes the posterior density function $P(\lambda / y)$ or equivalently the $\log$ of $P(\lambda / y)$. Given a suitable prior $P(\lambda)$, MAP-reconstruction can be formulated as,

$\lambda=\max _{\lambda \geq 0}[\log P(y / \lambda)+\log P(\lambda)]$

The image field is assumed as Markov random field (MRF) [4] and by Hammerseley-Clifford theorem [11], image $\lambda$ is characterized by Gibbs distribution,

$$
p(\lambda)=\frac{1}{Z} \exp \left(-\frac{1}{\beta} \sum_{n} \sum_{l \in Q_{n}} w_{n l} V\left(\lambda_{n}-\lambda_{l}\right)\right)
$$

where, $Z$ is the normalizing constant for the distribution, $\beta$ is the Gibbs hyper-parameter, $w_{n l}$ is the weight of pixel $l \varepsilon Q_{n}[3], Q_{n}$ is the nearest neighbor set of pixel $n$ and $V\left(\lambda_{n}, \lambda_{l}\right)$ is termed as the potential at site $n$ due to the nearest neighbor element $l$.

The equation (2) is difficult to solve due to the complicated nature of the prior. Green [3] has proposed one step late (OSL) approximation for an iterative update to the MAP-problem and is given by,

$$
\begin{aligned}
\lambda_{n}^{k+1} & =\frac{\lambda_{n}^{k}}{\sum_{m=1}^{M} p_{n m}+\left.\frac{1}{\beta} \sum_{l \varepsilon Q_{n}} w_{n l} \frac{\partial\left(V\left(\lambda_{n}, \lambda_{l}\right)\right)}{\partial \lambda_{n}}\right|_{\lambda_{n}=\lambda_{n}^{k}}} \\
& \times \sum_{m=1}^{M} \frac{y_{m} p_{n m}}{\sum_{o=1}^{N} \lambda_{o} p_{o m}}
\end{aligned}
$$

It should be noted that, MAP estimate given by eqn. (4) tends to ML estimate when the prior distribution tends to uniform distribution. This is understandable because the uniform distributed prior gives equal probability for any estimate to be a solution.

The next step is the proper modeling of the interacting potential $V\left(\lambda_{n}, \lambda_{l}\right)$ between the pixel at site $n$ and its neighbors $l \varepsilon N_{n}$. A large number of potentials (see $[3,4,5]$ ) have been suggested in literature to produce desired image characteristics. It is a general nature of these potentials function to smooth the edges irrespective of the density class, producing over-smooth reconstruction.

\section{Proposed Potential Function}

The previous section has highlighted the importance of pixel-pixel interacting potential function. A fuzzy logic based potential is proposed for edge-preserving reconstructed images. This consists of two basic operations: fuzzy filtering followed by fuzzy smoothing. Fuzzy derivatives are used for the detection of edges in the nearest neighborhood window (which is equivalent to recognizing nearby density classes). Fuzzy smoothing penalizes those pixels for which no edge is detected in the neighborhood. The proposed fuzzy logic is borrowed from Ville et. al. who used it for image restoration [9]. We have extended the fuzzy algorithm to suit image reconstruction in PET. Nevertheless the idea is expanded, improved and adapted for PET image reconstruction.

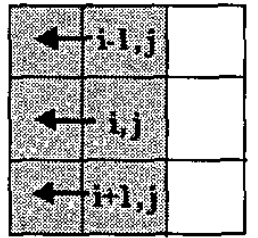

(a)

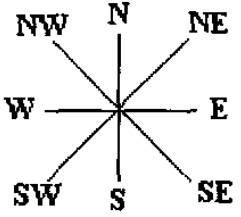

(b)
Figure 1. $3 \times 3$ neighborhood of a central pixel $(i, j)$, showing the directional derivative along $\hat{W}$.

\subsection{Fuzzy Derivatives for Edge Detection}

Consider a $3 \times 3$ neighborhood window of a pixel $(i, j)$ as shown in Fig.1. A simple derivative at central pixel $(i, j)$ in the direction $\mathrm{D}(\mathrm{D} \varepsilon \operatorname{dir}, \operatorname{dir}=\mathrm{N}, \mathrm{S}, \mathrm{E}, \mathrm{W}, \mathrm{NE}, \mathrm{NW}, \mathrm{SE}, \mathrm{SW})$ is defined as the difference between the pixel at $(i, j)$ and its neighbor in the direction $\mathrm{D}$ and is defined by $\nabla(i, j) \hat{D}$. At $\mathrm{k}^{\text {th }}$-iteration, the derivative $\nabla^{k}(i, j)$ is defined as,

$$
\nabla^{k}(i, j) \hat{n}=\left|\lambda^{k}(*, *)-\lambda^{k}(i, j)\right| \hat{n}
$$

where, $\lambda^{k}(i, j)$ represents the pixel value at $(i, j)$ for iteration $k$ and $\lambda^{k}(*, *)$ is the pixel intensity at the immediate neighboring location in the direction $\hat{n}$.

Consider an edge passing through the neighborhood of pixel $(\mathrm{i}, \mathrm{j})$ in the direction North-South. The value of the derivative $\nabla^{k}(i, j) \hat{E}$ will be large. In addition to $\nabla^{k}(i, j) \hat{E}$ being large, the derivative of the neighboring pixels perpendicular to direction of the edge, $\nabla^{k}(i-1, j) \hat{E}$ and $\nabla^{k}(i+1, j) \hat{E}$ should also be large, if there is an edge passing through $(i, j)$ (see Fig. 1). The reasoning is based on the observation that a small fuzzy derivative most likely is caused by noise, while a large fuzzy derivative is caused by an edge in the image. Therefore, if two out of three derivative values are small, it is safe to assume that no edge is present at the location $(i, j)$. In such a case, penalization is carried out on the pixel $(\mathrm{i}, \mathrm{j})$ to remove noise. 
For identifying edge in a particular direction, three elementary derivatives are chosen. For example, to detect an edge along $\hat{E}$ direction, the derivatives are: $\nabla^{k}(i, j) \hat{E}$, $\nabla^{k}(i-1, j) \hat{E}$ and $\nabla^{k}(i+1, j) \hat{E}$ for a $3 \times 3$ window. For $\hat{D}=\hat{E}$ the fuzzy derivative is defined as follows:

$$
\text { If }\left\{\begin{array}{l}
\nabla^{k}(i, j) \text { and } \nabla^{k}(i-1, j) \text { are small or } \\
\nabla^{k}(i, j) \text { and } \nabla^{k}(i+1, j) \text { are small or } \\
\nabla^{k}(i-1, j) \text { and } \nabla^{k}(i+1, j) \text { are small } \\
\text { then, } \nabla_{F}^{k}(i, j) \hat{W} \text { is small }
\end{array}\right.
$$

$$
\text { Else, } \nabla_{F}^{k}(i, j) \hat{W} \text { is l arge }
$$

Similarly, fuzzy derivatives $\nabla_{F}^{k}(i, j) \hat{D}$ are defined for all directions i.e, $\{E, N, S, N W, S W, N E, S E\}$.

\subsection{Membership Function}

We make use of fuzzy set small to compute the value that expresses the degree to which the fuzzy derivative is small. Membership function for the property small for site (i,j) along the direction $\hat{E}$ at $k^{\text {th }}$ iteration is defined as,

$$
M^{k}(i, j) \hat{E}= \begin{cases}\text { small, } & \text { if } \nabla^{k}(i, j) \leq \nabla_{M}^{k}(i, j) \\ \text { large, } & \text { otherwise }\end{cases}
$$

where,

$$
\nabla_{M}^{k}(i, j) \hat{E}=\operatorname{median}\left\{\nabla^{k}(i-1, j), \nabla^{k}(i, j), \nabla^{k}(i+1, j)\right\}
$$

Due to the iterative nature of the MAP algorithm, the proposed membership function small gets updated with each iteration. Similarly membership function is defined for all the derivatives $\nabla_{F}^{k}(i, j) \hat{D}$ along all the directions viz. $\{E, N, S, N W, S W, N E, S E\}$.

\subsection{Fuzzy Penalization}

The final step in the computation of fuzzy filter is the defuzzification. We are interested in obtaining correction term $\Delta^{k}(i, j)$, which can be added to pixel value at location $(i, j)$. The idea is to cancel out the effect of one derivative value which turns out to be high due to noise. When a particular derivative is large, and the neighboring derivatives are also large, then there is an edge and hence no panelization has to be carried out for that pixel. On the other hand, when a particular derivative is large, and the neighboring derivatives are not large, then the large value of the derivative could be due to noise. The effect of noise is removed by penalizing the pixel based on the derivative values in all other directions. The following rule is used for penalization :

$$
\text { if } \begin{gathered}
\nabla_{F}^{k}(i, j) \hat{D} \text { is small, then } \Delta^{k}(i, j) \hat{D}=\nabla_{F}^{k}(i, j) \hat{D} \\
\text { else, } \Delta^{k}(i, j) \hat{D}=0
\end{gathered}
$$

where, $\Delta^{k}(i, j) \hat{D}$ is the feedback at site $(i, j)$ due to the adjacent pixel in the direction $\hat{D}$. Eight such rules are used to get the contribution from all the eight directions. Hence, the total correction term $\Delta_{T}^{k}(i, j)$ for pixel at $(i, j)$ considering all the directions after $k^{\text {th }}$ iteration is given by,

$\Delta_{T}^{k}(i, j)=\frac{1}{8} \sum_{\hat{D}} \Delta^{k}(i, j) \hat{D}$

Replacing the error term in eqn.(4),
$\left.\sum \sum_{l \varepsilon Q_{n}} w_{n l} \frac{\partial\left(V\left(\lambda_{n}, \lambda_{l}\right)\right)}{\partial \lambda_{n}}\right|_{\lambda_{n}=\lambda_{n}^{k}}$ by the proposed correction term $\Delta_{T}^{k}(n)$, the OSL-algorithm gets modified to,

$$
\lambda_{n}^{k+1}=\frac{\lambda_{n}^{k}}{\sum_{m=1}^{M} p_{n m}+\frac{1}{\beta} \Delta_{T}^{k}(n)} \sum_{m=1}^{M} \frac{y_{m} p_{n m}}{\sum_{o=1}^{N} \lambda_{o} p_{o m}}
$$

where, coordinates $(i, j)$ in the correction term is replaced by a single coordinate $n$ where, $n=(i-1) \sqrt{n}+j$. In the iterative image reconstruction procedure, the final correction term is fed back to update the pixel after each iteration. The iterations are continued until acceptable convergence is obtained.

Fuzzy rules are also extended for $5 \times 5$ neighborhood window to study the effect of window size on the image quality. The sensitivity of edge detection depends upon the number of derivatives used for edge detection. To enhance the detectivity of edges, five elementary derivatives per direction are taken. 3:5 rule is used for edge detection. The membership function will have the same form except that the median is taken over all the five elementary derivatives. Fuzzy derivative is calculated using five elementary derivatives per direction. The rest of the method is similar to that for $3 \times 3$ window.

\section{Proposed Potential Function}

\subsection{Simulated PET System}

The algorithm has been tested on a simulated PET system. The PET system consists of a ring detector with 64 detectors and the object space is decomposed into $64 \times 64$ square pixels. An electron-positron annihilation event 
occurring inside a pixel is governed by a number of physical phenomena such as attenuation, scattering, absorption and detector characteristics. All these physical processes have a bearing on the probability matrix. In this study, we assume that the probability of an emission in box $n$ and its detection in tube $m$ depends only on the geometry of the measurement system. In such a case, an annihilation event in box $n$ is detected in a tube $m$ with the probability $p_{n m}$ proportional to the angle of view from the center of the box $n$ in to the detector tube $m$, i.e, $p_{n m}=\theta_{n m} / \pi$. Shepp et. al. [2] have shown that the choice of $p_{m m}$ based only on the geometry of the measurement system is reasonable, and that the results of the reconstruction do not depend critically on the choice of $p_{n m}$. Before the reconstruction begins, the probability matrix $\mathbf{P}=\left[p_{n m}\right], n=1, \ldots, N$ and $m=1, \ldots, M$ is precomputed and stored.

For simulating measurement data, the well known Monte Carlo procedure is used [1]. The mathematical phantom used is made up of nine elliptic objects having different sizes, orientations and density values. The image of the phantom is shown in Fig. 3(a). We used a source image with 100,000 emission counts.

\subsection{Algorithm Evaluation}

The proposed algorithm has been studied with $3 \times 3$ and $5 \times 5$ neighborhood windows. The images reconstructed using the proposed fuzzy method are compared with images reconstructed by MRP and MAP methods. The MAPalgorithm with potential $V_{1}=\sum_{l E N_{n}}\left(\lambda_{n}-\lambda_{l}\right)^{2}$ with $\beta=2.5 \times 10^{4}$ is used in this study. It has been found that $\beta=2.5 \times 10^{4}$ gives best estimate. The performances of the proposed algorithms are evaluated using two different image-based quantitative criteria as given below:

Residual Error - This measures the deviation of the generated pseudo-projections of the reconstructed image from the observed projection data $y_{m}$. Residual error $\rho\left(\lambda^{k}\right)$ at $k^{\text {th }}$ iteration is given by,

$$
\rho\left(\lambda^{k}\right)=\sum_{m=1}^{M}\left(y_{m}-\sum_{n=1}^{N} \lambda_{n}^{k} p_{n m}\right)^{2}
$$

Fig. 2 plots the residual error of the reconstructed images using the proposed algorithm (with $3 \times 3$ and $5 \times 5$ window), MAP and MRP algorithms. From these plots it is clear that the proposed algorithm has the lowest residual error compared to MAP and MRP algorithms. Particularly, the proposed algorithm with $5 \times 5$ window has produced the lowest residual error. This is because $5 \times 5$ window enhances the detectivity of edges.

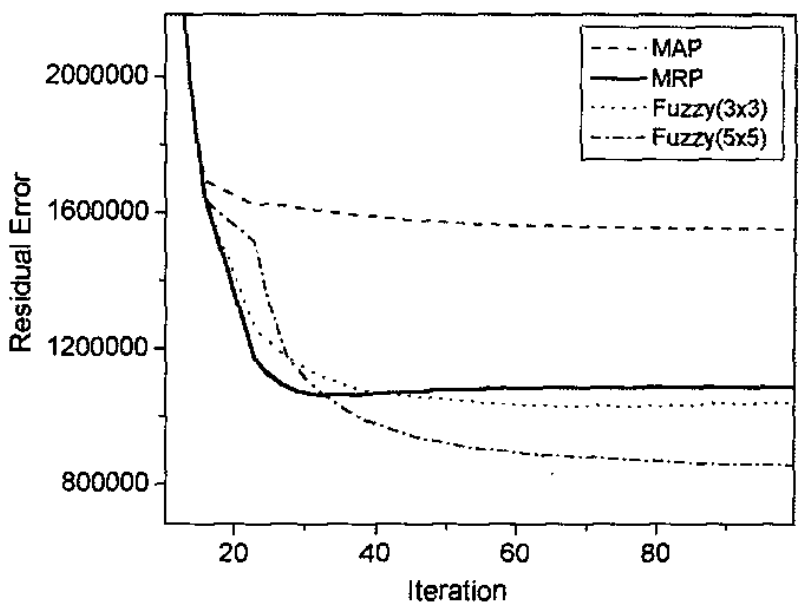

Figure 2: Residual Error Plot for MAP, MRP and proposed fuzzy algorithm with $3 \times 3$ and $5 \times 5$ window.

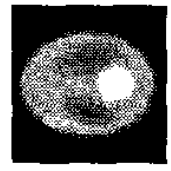

(a)

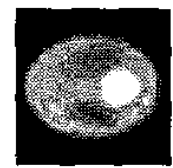

(e)

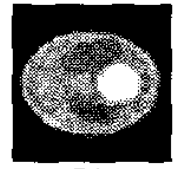

(b)

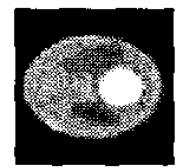

(f)

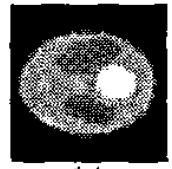

(c)

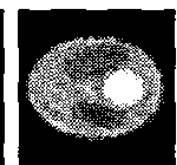

(g)

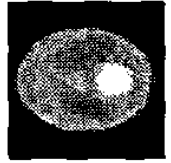

(d)

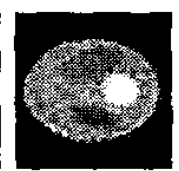

(h)

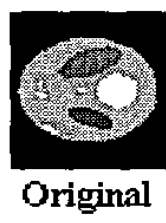

Figure 3: (a), (b), (c), (d) and (e), (f), (g), (h) are the reconstruction using MAP, MRP, proposed algorithm with $3 \times 3$ and $5 \times 5$ window after 50 and 100 iterations respectively. For comparison original phantom is also shown.

Visual Inspection - Final and the most important test is the visual inspection of the reconstructed images. The reconstructed images using MAP, MRP, and proposed algorithm (for $3 \times 3$ and $5 \times 5$ ) after 50 and 100 iterations respectively are shown in Fig. 3. For quality assessment, the original test phantom is also shown in Fig. 3. The images reconstructed using the proposed algorithm ( Fig. 3(c),(g),(d),(h)) are more appealing and rich in edges compared to those reconstructed using MAP ( Fig. 3(a),(e)) and MRP (see fig. 3(b),(f)) algorithms. 
It is evident from the reconstructed images that MAP over smooths the reconstruction while MRP is capable of preserving small details at the cost of streaking effect [7]. The images reconstructed by the proposed algorithm preserve the edges and the finer details, and are not over smoothened.

\section{Conclusions}

We have presented a new approach for better edge preserving reconstruction for PET modality. This is based on the application of fuzzy rule based techniques to model the potential (which accounts for the nearest neighbor interaction) in the image reconstruction problem. Two basic steps are performed namely fuzzy filtering and fuzzy smoothing. Fuzzy filtering is used for the detection of edges in the reconstruction while fuzzy smoothing is used to penalize only those pixels for which the edges are absent in the nearest neighborhood. These operation are continued iteratively until acceptable convergence is obtained. The computer simulated experimental PET studies show that the proposed technique is promising. It is found that residual error is low for the proposed algorithm.

\section{Acknowledgement}

Thanks are due to Council of Scientific and Industrial Research for Junior Research Fellowship to the first author. First author declares that this work is partly supported by Council of Scientific and Industrial Research, New Delhi, India.

\section{References}

[1] Y. Vardi, L. A. Shepp, and L. Kaufmann, "A statistical model for positron emission tomography ", J. Amer. Stat. Assoc., Vol. 80, pp. 8-37, 1985.

[2] L.A. Shepp and Y. Vardi., " Maximum likelihood estimation for emission tomography ", IEEE Trans. on Med. Img ., MI-1: pp. 113-121, 1982.

[3] P.J. Green, "Bayesian reconstruction from emission tomography data using a modified EM algorithm", IEEE Trans. on Med. Img., Vol.9, No.1, March, 1990.

[4] Z. Zhou, R. M. Leahy and J. Qi, "Approximate maximum likelihood hyperparameter estimation for Gibbs prior ", IEEE Trans. on Img. proc., Vol.6, No.6, pp.844861, June, 1997.

[5] J. Nuyts, D. Bequé, P. Dupont, and L. Mortelmans, "A Concave Prior Penalizing Relative Differences for Maximum-a-Posteriori Reconstruction in Emission Tomography ", IEEE Trans. on Nucl. Sci., Vol. 49, No. 1, pp. $56-60$, Feb. 2002.
[6] S. Alenius and U. Ruotsalainen, "Using Local Median as the Location of Prior Distribution in Iterative Emission Tomography Reconstruction ", IEEE Tran. Nucl. Sci., Vol.45, No.6, Dec. 1998.

[7] S. Alenius and U. Ruotsalainen, "Generalization of Median Root Prior Reconstruction", IEEE Trans. Med. Img., Vol.21, No.11, Nov., 2002.

[8] E. Veclerov and J. Llacer, "Stopping rule for MLE algorithm based on statistical hypothesis testing ", IEEE Trans. on Med. Img., MI-6, pp. 313-319, 1987.

[9] D. Van De Ville, M. Nachtegael, D. V. Weken, E. E. Kerre, W. Philips and I. Lemahieu, "Noise Reduction by Fuzzy Image Filtering ", IEEE Trans. Fuzzy Sys., Vol. 11, NO. 4, Aug. 2003.

[10] D. Van De Ville, W. Philips, and I. Lemahieu, Fuzzy Techniques in Image Processing. New York: SpringerVerlag, 2000, vol. 52, Studies in Fuzziness and Soft Computing, ch. Fuzzy-based motion detection and its application to de-interlacing, pp. 337-369.

[11] J. Besag , " Spatial interaction and the statistical analysis of lattice systems ", Jl. of Royal Stat. Soc. B, Vol.36, pp. 192-236, 1974.

[12] N. Rajeevan, K. Rajgopal and G. Krishna. " VectorExtrapolated fast maximum likelihood estimation algorithms for emission tomography ", IEEE Trans. on Med. Img., Vol. 11, No. 1, March 1992.

[13] L. Kaufmann, "Implementing and accelerating the EM-algorithm for positron emission tomography ",IEEE Trans. Med. Img., Vol. MI-6, pp.37-51, Mar. 1987. 